\title{
REVIEW ARTICLE OPEN Skeletal muscle stem cells in comfort and stress
}

\author{
Brendan Evano ${ }^{1,2}$ and Shahragim Tajbakhsh ${ }^{1,2}$
}

Investigations on developmental and regenerative myogenesis have led to major advances in decrypting stem cell properties and potential, as well as their interactions within the evolving niche. As a consequence, regenerative myogenesis has provided a forum to investigate intrinsic regulators of stem cell properties as well as extrinsic factors, including stromal cells, during normal growth and following injury and disease. Here we review some of the latest advances in the field that have exposed fundamental processes including regulation of stress following trauma and ageing, senescence, DNA damage control and modes of symmetric and asymmetric cell divisions. Recent studies have begun to explore the nature of the niche that is distinct in different muscle groups, and that is altered from prenatal to postnatal stages, and during ageing. We also discuss heterogeneities among muscle stem cells and how distinct properties within the quiescent and proliferating cell states might impact on homoeostasis and regeneration. Interestingly, cellular quiescence, which was thought to be a passive cell state, is regulated by multiple mechanisms, many of which are deregulated in various contexts including ageing. These and other factors including metabolic activity and genetic background can impact on the efficiency of muscle regeneration.

npj Regenerative Medicine (2018)3:24; https://doi.org/10.1038/s41536-018-0062-3

\section{INTRODUCTION}

Regenerative myogenesis has emerged as arguably one of the most powerful paradigms to investigate a variety of processes involving stem cells and tissuegenesis. Adult skeletal muscle satellite (stem) cells emerge from a proliferative population of myogenic cells that reversibly exit the cell cycle asynchronously during perinatal growth. ${ }^{1,2}$ They are located between muscle fibres and the basement membrane ensheathing it and since their initial identification in the frog, ${ }^{3}$ genetic and cell lineage strategies led to detailed analysis of their properties. Notably, critical regulators of quiescence, commitment and self-renewal have been identified, while exposing underlying heterogeneities in myogenic cell states. ${ }^{1,4}$

In vertebrates, genetic and embryological studies have shown that the bHLH myogenic regulatory factors (MRFs) Myf5, Mrf4, Myod and Myogenin have crucial roles in governing striated muscle cell fate and differentiation. Mice triple mutant for Myf5, Myod and Mrf4 lack skeletal muscles and their progenitors pointing to these genes as critical determination factors, whereas Myogenin and Mrf4 act during differentiation. ${ }^{4-7}$ In the adult, Myf5 is expressed in quiescent and activated satellite cells, ${ }^{8}$ whereas MYOD protein expression is a hallmark of an activated satellite cell. ${ }^{4-6}$ Upstream transcription factors include Pax3, Tbx 1 , Six $1 / 4$ and Pitx2 and they act in different locations in the embryo to establish the founder muscle stem cell population. ${ }^{9}$ The properties of the paraxial mesoderm from which head and body muscles arise are also different. All body muscles and some located in the head arise from transient structures called somites, and these are under the regulation of the paired/homeobox transcription factors Pax3 in the embryo, and later, Pax $7 .{ }^{10,11}$ In contrast, head muscles are derived from cranial paraxial mesoderm and are Pax3independent, but regulated in the embryo by $T b x 1$ and Pitx2 among other genes. ${ }^{9,12}$ From mid-embryonic stages, virtually all stem/progenitor cells throughout the body are marked by Pax 7 expression. These cell-intrinsic differences observed during embryogenesis occur in the context of a heterogeneous extrinsic milieu. Indeed, an important consideration is the role of stromal cells that constitute the stem cell niche, and that also arise from distinct embryological origins in the head and the body. Their impact on muscle stem/progenitor cell fates remains largely unexplored. Some of these issues will be discussed in this review.

\section{THE INTERPLAY BETWEEN SATELLITE AND STROMAL CELLS IN SKELETAL MUSCLE}

Although fewer studies have focused on the role of interstitial cells (Fig. 1), their critical roles in homoeostasis and regeneration has been highlighted in several reports. For example, fibroadipogenic progenitors (FAPs) promote myoblast differentiation and participate in fibrosis following muscle damage. ${ }^{13,14}$ Another cell type, called PICs (Pw1 + interstitial cell) was also identified as residing outside the basement membrane of the muscle fibre. Pw1 is an imprinted gene that is involved in stress regulation. ${ }^{15,16}$ The transplantation of PICs into injured muscle results in their contribution to regenerating fibres. ${ }^{16}$ Mesoangioblasts that are associated with blood vessels were also reported to contribute to skeletal muscles. ${ }^{17}$ Interestingly, mesoangioblasts isolated from mouse, dog and human express high levels of Pw1, where this gene was shown to confer the myogenic potential of mesoangioblasts, and their ability to cross the vessel wall. ${ }^{18}$ Recently, an interstitial cell type that is marked by the basic-Helix-loop-Helix transcription factor Twist2 (Dermo1), was reported to be Pax7negative during homoeostasis and following muscle injury. ${ }^{19}$ Intriguingly, these cells contribute specifically to type $\mathrm{llb} / \mathrm{x}$ myofibres during adulthood and muscle regeneration, and their genetic ablation causes wasting of type Ilb (fast glycolytic) myofibres. ${ }^{19}$ How these different cell types are related to socalled "mesenchymal stem cells" remains obscure. FAPs can be

\footnotetext{
${ }^{1}$ Stem Cells and Development, Department of Developmental \& Stem Cell Biology, Institut Pasteur, 75015 Paris, France and ${ }^{2}$ CNRS UMR 3738 , Institut Pasteur, 75015 Paris, France Correspondence: Shahragim Tajbakhsh (shahragim.tajbakhsh@pasteur.fr)
}

Received: 25 July 2017 Accepted: 28 November 2018

Published online: 21 December 2018 


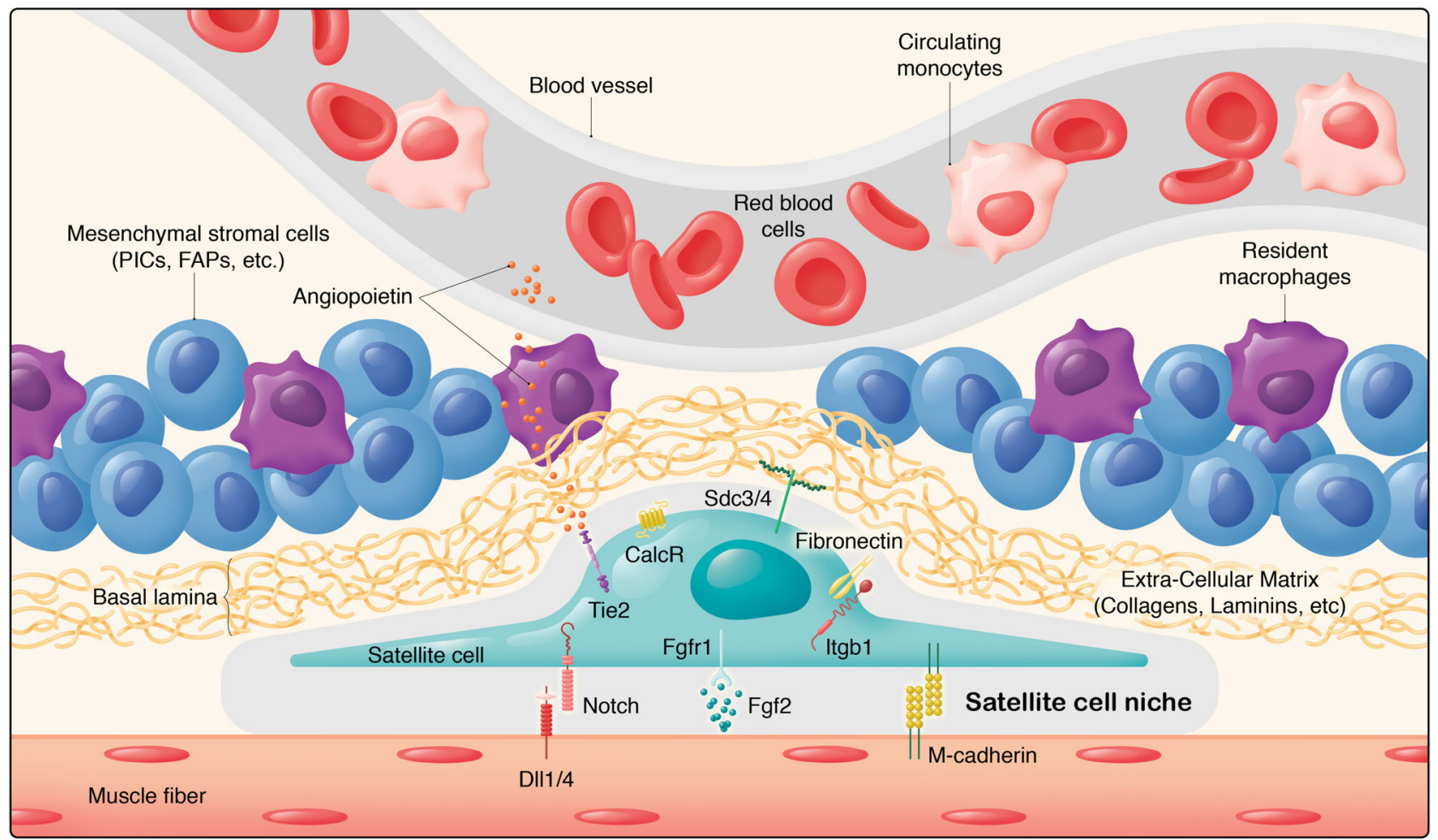

Fig. 1 The satellite cell and stromal cell niche. Satellite cells states are regulated through their interactions with their microenvironment. While direct interactions (M-cadherin, Notch pathway) ${ }^{38,46}$ and communication (FGF2-FGFR1 pathway) $)^{48}$ between muscle fibres and satellite cells have been identified, muscle stem cells also interact with a variety of components of the extracellular matrix (e.g. Collagens VI and V, Laminin, Fibronectin, SDC3/4) (5,79 and diffusable cytokines and growth factors (e.g. Angiopoietin-Tie2 receptor) ${ }^{37}$. In addition to satellite cells, several cell types contribute to muscle growth, homoeostasis and regeneration, including pericytes, mesenchymal stromal cells (e.g. Pw1+ Interstitial Cells, FibroAdipogenic Progenitors, Twist $2+$ cells) ${ }^{16,19,21}$, immune cells (e.g. resident or infiltrating macrophages) ${ }^{156}$ as well as connective tissue cells. These interactions are remodelled during ageing, notably with increased FGF2 production from muscle fibres and decreased expression of FGFR1 in satellite cells, driving satellite cells to break quiescence ${ }^{39}$, and decreased levels of fibronectin ${ }^{45}$, which weakens satellite cell adhesion capacity and increases their susceptibility to apoptosis by anoikis

isolated by cytometry using PDGFRa/Sca1 ${ }^{20}$ whereas PICs (PW1 +/PDGFRa- fraction with myogenic capacity) were reported to be a sub-population of interstitial cells. ${ }^{21}$ As the field tries to resolve these different cell types further, it is interesting to note that a detailed study of mesenchymal "stem" cells pointed to a substantial heterogeneity in this population depending on their tissue of origin, ${ }^{22}$ therefore a concerted effort is clearly needed to further characterise stromal cells in different tissues and address the potentially misleading designation of stromal cells generically as "mesenchymal stem cells". Given these findings, and the potential misappropriation of cell populations, single cell mass spectrometry and single cell RNAseq of the entire muscle resident cell population was done to resolve some of these discrepancies. ${ }^{23}$ Of note a total of 10 different cell types were identified, including known populations (satellite cells, FAPs, endothelial cells, etc) and previously uncharacterised resident tenocyte-like cells and smooth muscle/mesenchymal cells with myogenic potential were identified. $^{23}$

More detailed studies on FAPs have shown that TNFa-mediated apoptosis of this population is critical for normal regeneration. During chronic injury, as with dystrophic $m d x$ mice, continued expression of transforming growth factor $\beta 1$ (TGF $\beta 1$ ) results in persistence of FAPs and fibrosis. Pharmacological inhibition with the tyrosine kinase inhibitor Nilotinib, which has potent antifibrotic activity, blocks TGF $\beta 1$ activity and results in reduced fibrosis. ${ }^{20}$ However, Nilotinib treatment also blocks expansion of FAPs and compromises regeneration through a non-cellautonomous anti-proliferative effect on satellite cells. ${ }^{24}$ These studies and others cited below highlight the dynamic nature of regeneration, and the importance of determining when to intervene for a desired outcome.

Stromal cells that have myogenic capacity have been considerably less well characterised compared to satellite cells, and their contributions to muscle or self-renewal into a satellite cell position are limited, or not demonstrated, compared to bona fide satellite cells. Significantly, elimination of satellite cells by selective diphtheria toxin ablation results in failed regeneration, ${ }^{25-27}$ indicating that in the short term, non-satellite cells do not contribute to muscle regeneration. Similar ablation studies should be extended to all interstitial cell types.

It is interesting to note that satellite cells have significantly distinct genetic requirements in different anatomical locations as indicated above (e.g. Tbx1/Pitx2 in head; Pax3 in body). It is therefore likely that stromal cells, which can be of mesodermal or neural crest origin, might impact differentially on the fate of myogenic cells in relation to their anatomical location. ${ }^{4}$ Importantly, emerging satellite cells continue to proliferate until about 2 weeks postnatally, yet they are ensheathed under a basement membrane from mid-late foetal stages. ${ }^{1,28}$ Therefore, contact with extracellular matrix proteins in the basal lamina of the basement membrane is not sufficient to trigger cell cycle exit. In addition, enseathment under the basal lamina results in pre-quiescent and post-quiescent satellite cells being physically separated from stromal cells and in contact only with the myofibre, until its disruption following injury (Fig. 2). How sporadic interactions with stromal cells prior to this confinement affect the fate of myogenic cells is an open question. Given that the transcriptome profiles of Pax7+ myogenic cells change significantly during prenatal and 


\section{Skeletal muscle niches}

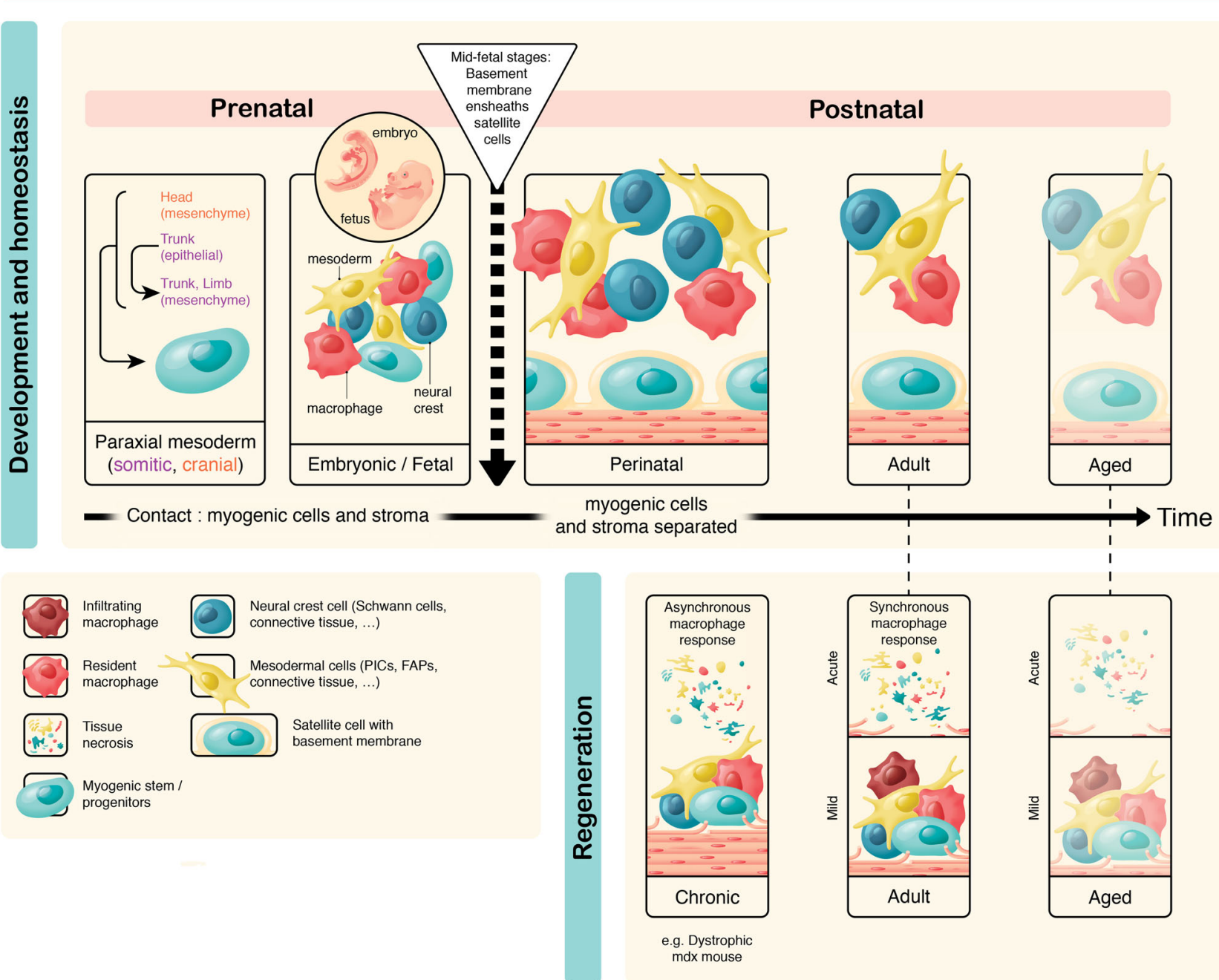

Fig. 2 Developmental, adult, ageing and diseased skeletal muscle niches. During development and regeneration, stromal and myogenics cells can be in direct contact, and myogenic cells can be exposed to stromal-derived extracellular matrix proteins, whereas in the late foetal to postnatal stages, muscles stem cells are separated from stromal cells by a basement membrane (from mid-foetal stages) ${ }^{1,28}$. Stromal cells have distinct embryonic origins depending on their anatomical location-those in the head are of neural crest and mesoderm origin, whereas those in the limbs are mostly of mesodermal origin ${ }^{4}$. In addition to this spatial character, niche cells evolve during development and postnatal life thereby introducing a temporal dimension to the regulation of muscle stem cells as they give rise to quiescent satellite cells and age. The postnatal niche is disrupted following chronic (e.g. myopathies) and acute (chemical, physical) injury. In the former, there is an asynchronous response of infiltrate and in the latter, more phasic appearance and disappearance of neutrophils and macrophages is noted

postnatal development, ${ }^{29-31}$ stem cell and niche cell interactions need to be explored further in different contexts when direct satellite and niche cell contacts can occur (Fig. 2).

\section{IDENTIFICATION OF THE QUIESCENT STATE}

Non-cycling cells have been characterised in different states: quiescent, terminally differentiated, apoptotic/necrotic, and senescent. In contrast to apoptosis, differentiation and senescencecellular quiescence is a reversible cell state representing a noncycling cell. In multicellular organisms, postnatal tissue homoeostasis and regeneration upon trauma are mediated by adult tissue specific stem cells that are generally quiescent. Following injury, and in response to diffusible and mechanical cues, adult satellite cells activate to generate transit amplifying myoblasts that will in turn fuse to form multinucleated myofibres. A subset of the transit amplifying precursors renews the stem cell pool by returning to the quiescent state. In vitro models can recapitulate this process to some extent where a pool of 'reserve' cells is associated with differentiated myotubes. ${ }^{32}$ Efforts have also been made to artificially recapitulate cellular quiescence or the niche environment ex vivo. ${ }^{33-35}$

In the yeast Saccharomyces cerevisiae, quiescence is induced in response to nutrient limitation. ${ }^{36}$ In multicellular organisms, cellular quiescence might also act as a protective mechanism, against accumulation of mutations due to proliferation, but also against various environmental stresses. Deregulation of quiescence can lead to precocious differentiation, senescence, or apoptosis, and it is associated with impaired tissue regeneration. ${ }^{37-51}$ In the context of therapies, a promising strategy is the transplantation of tissue stem cells that have been corrected in vitro. To date, a major challenge has been overcoming the loss of stemness properties following ex vivo expansion, ${ }^{52,53}$ and diverse strategies, ${ }^{54-56}$ including isolation of transplantable myogenic cells from teratomas, ${ }^{57}$ show some promising results. Furthermore, retaining quiescence properties might be a means to 


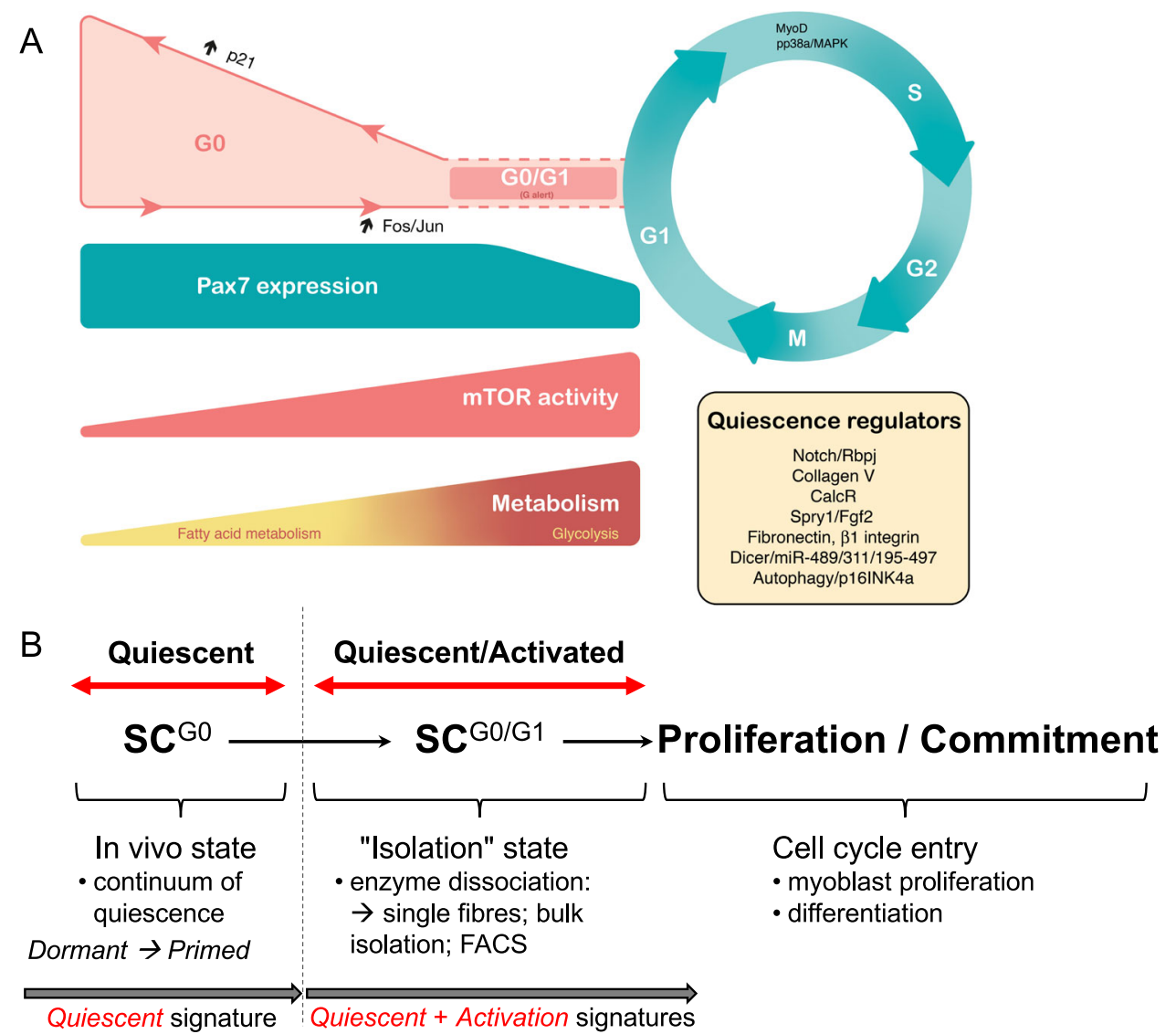

Fig. 3 Quiescence to proliferation transition in satellite cells. a During homoeostasis, adult satellite cells are maintained in a reversible nonproliferating quiescent G0 state by regulators including Calcitonin receptor, Collagen V, Notch pathway, FGF signalling and effectors of the RNAi machinery ${ }^{39,40,46,51,79}$. However, specific quiescence markers are still lacking. Satellite cells within a healthy tissue respond to a distant injury by transiting from deep quiescence to a quiescent G0/G1 or ' $G\left(\right.$ alert)' state ${ }^{85}$, with increased proliferative capacity and regenerative potential. This transition is under the control of mammalian target of rapamycin (mTOR) signalling, which in turns controls mitochondrial metabolism (see text). Following an acute tissue injury or chronic mild degeneration of muscle fibres, satellite cells exit from their quiescent state and proliferate. This transition is accompanied by a metabolic shift from fatty acid oxidation to glycolysis. ${ }^{147}$ Some cells irreversibly exit the cell cycle to differentiate into mononuclear myocytes that eventually fuse to regenerate muscle fibres, while others self-renew and return to quiescence. Entry into quiescence is poorly characterised, and an activation marker is MYOD. b Isolation of adult quiescent satellite cells involves repeated mechanical and enzymatic dissociation of the tissue. These procedures invariably lead to satellite cell activation, as shown by the rapid upregulation of FOS and JUN, and phosphorylation of p38 ${ }^{65}$

survive the transplantation process, ${ }^{58,59}$ thereby underscoring the importance of characterising this cell state.

Cellular quiescence, or G0, is defined as a transient and reversible cell cycle arrest during the G1 phase (Fig. 3). It is characterised by a decrease in cell size, increased nucleocytoplasmic ratio, $2 \mathrm{~N}$ genome content, low RNA and protein synthesis, altered metabolism and gene expression profile. ${ }^{60}$ Quiescence had been considered for some time to be a default state corresponding to the absence of proliferation and differentiation. It is now clear that cellular quiescence is an actively maintained state ${ }^{61}$ with new functional markers continually being identified.

Considerable effort has been put into identifying quiescencespecific markers, notably for satellite cells ${ }^{31,41,45,62-67}$ (Fig. 3a). Of those, Calcr (Calcitonin receptor) and Odz4 are of interest as they encode membrane-associated proteins that could potentially be used to isolate quiescent muscle stem cells by fluorescence activated cell sorting (FACS). ${ }^{68,69}$ Interestingly, Odz4 is reexpressed earlier than Calcr in satellite cells during regeneration, ${ }^{69}$ and proliferating neonatal satellite cells express Odz4, but not Calcr, indicating that some quiescence genes are differentially expressed during homoeostasis, muscle regeneration and postnatal growth.

Until recently, studies focused on identifying quiescencespecific transcripts in adult muscle stem cells have required purification procedures based on mechanical and enzymatic dissociation followed by satellite cell isolation by FACS. These involve dissociating satellite cells from their microenvironment, which invariably leads to their activation (Fig. 3b). In addition, it is now clear that satellite cell activation occurs more rapidly than previously thought; for example phosphorylated p38 appears within 30 min after isolating satellite cells on myofibres. ${ }^{70}$ Hence, some highly dynamic quiescence-specific or activation-specific genes, non-coding RNAs, and epigenetic states might have been missed in previous studies ${ }^{31,65,67}$ (Fig. 3b). Accordingly, it has been known for decades that the Fos/Jun stress response pathway activates within minutes during the $\mathrm{G} 0$ to $\mathrm{G} 1$ transition or in response to stress ${ }^{31,71}$ (Fig. 3b). Although some markers allow the identification of deeply quiescent satellite cells and of actively proliferating myoblasts, in vivo dynamic markers to follow early activation or re-entry into quiescence during regeneration are largely lacking.

\section{QUIESCENT MUSCLE STEM CELLS}

Molecular regulation of quiescence

Notch signalling has a critical role in maintaining quiescence of satellite cells, and the expression of canonical Notch targets (e.g. 
Hes, Hey) is markedly reduced before cells enter the cell cycle, thereby allowing MYOD protein to accumulate. ${ }^{20,28}$ Later during regeneration, Notch signalling increases, both in differentiating and in self-renewing myoblasts. ${ }^{72}$

During homoeostasis, quiescent satellite cells display high levels of Notch pathway activity, relayed by its intracellular effector $R b p j$. Satellite cell-specific deletion of $R b p j$ during homoeostasis results in loss of satellite cells, their differentiation, and fusion to existing myofibers. ${ }^{38,46}$ Intriguingly, in this case the majority of satellite cells boycott S-phase and do not enter the cell cycle (absence of BrdU uptake). Deletion of Rbpj also leads to an impaired regeneration capacity following injury. ${ }^{46}$ Nonetheless, Rbpj null satellite cells can undergo a normal activation and transit amplification after muscle injury, indicating different contextual roles for Notch signalling in maintenance of quiescence and initial cell cycle progression through G1/S in muscle homoeostasis or regeneration. ${ }^{72}$ Another role for Notch was proposed, this time through regulation of E3 ubiquitin ligase Mib1 (Mind bomb 1) which triggers endocytosis of ligands that interact with Notch receptor. ${ }^{73}$ Remarkably, sex hormones were reported to act during puberty, and after regeneration, to induce Mib1 in myofibres, thereby resulting in increased Notch activation in satellite cells, and their entry into quiescence. This model might appear counterintuitive, as it suggests that satellite cell quiescence in different muscle masses is under systemic regulation. Moreover, Notch activation inhibits expression of Myod and sustains the expression of Pax7 while allowing the proper homing of satellite cells to their niche, thereby participating in the maintenance of the non-committed state of satellite cells. ${ }^{46,74,75}$

As mentioned above, $\mathrm{Odz} 4$ is specifically expressed in neonatal and adult quiescent satellite cells. The ODZ proteins are type II transmembrane proteins, for which protein cleavage sites have been demonstrated in the extracellular and transmembrane domains, and cleaved intracellular ODZ2 acts as a transcription factor. ${ }^{76}$ Although the mode of action of Odz4 specifically in satellite cells remains to be determined, Odz4 germline null mice display a reduced body weight and size, decreased muscle mass and satellite cell pool size, both during homeostasis and after injury-mediated regeneration. Satellite cells isolated from these animals show prolonged in vitro proliferation and enhanced differentiation. $^{77}$

In addition, a satellite cell-specific deletion of Calcr sensitises satellite cells to apoptosis, which eventually decreases the size of the stem cell pool and impairs regeneration efficiency upon injury. ${ }^{51}$ Calcr encodes a G-protein coupled receptor (GPCR), known to regulate calcemia, and CALCR and its ligand calcitonin inhibit bone resorption in osteoclasts. ${ }^{78}$ In muscle, $\mathrm{Calcr}^{-1-}$ satellite cells express higher levels of the cell cycle marker KI67 and other cell-cycle-related genes (Ccna2, Ccnd1), without any increase in expression of myogenic genes such as Myod, and without impacting on myogenic differentiation. ${ }^{51}$ Interestingly, Calcr was reported to regulate satellite cell quiescence through CAMP-PKA signalling and thereby position satellite cells within their niche through CAMP-Epac signalling. ${ }^{51}$ Intriguingly, the ligand for CALCR, calcitonin, is produced by the thyroid, therefore, as for the regulation of Mib1 indicated above, this suggests that maintenance of satellite cell quiescence is also under systemic control. However recent evidence from our laboratory suggests that this may not be the case following identification of a novel Notch/ColV/Calcr axis where Collagen V, expressed by satellite cells, can act as a surrogate ligand for CALCR to maintain the quiescent state cell-autonomously. ${ }^{79}$

Interestingly, intron 4 of Calcr contains the microRNA miR489, which is necessary for their maintenance of the quiescent state by post-transcriptionally repressing the oncogene Dek. ${ }^{40}$ In addition, miR-195/497 was shown to induce cell cycle arrest ${ }^{80}$ suggesting that other miRs could have critical roles in maintenance of quiescence. Indeed, satellite cell loss of Dicer, which is a key mediator of miRNA processing, resulted in depletion of this pool, upregulation of $\mathrm{KI} 67$, and failure to regenerate following injury. ${ }^{40}$

Other signalling pathways have been explored in the context of satellite cell quiescence. For example, Angiopoietin 1 (ANG1) and its receptor TIE-2 regulate the quiescent state where TIE- 2 is expressed by a subset of quiescent satellite cells and ANG1/TIE2 signalling, through ERK $1 / 2$ pathway favours cell cycle exit. ${ }^{37}$ Furthermore, previous studies had suggested that c-MET and its ligand hepatocyte growth factor could regulate the quiescence to activation transition, however, conditional ablation of the c-Met receptor gene in satellite cells showed no requirement for satellite cell activation, myoblast proliferation, or myocyte differentiation, but a role in muscle fusion and regeneration. ${ }^{51}$ Oncostatin $M$, which belongs to the interleukin- 6 family of cytokines, also regulates satellite cell quiescence as depletion of its receptor leads to loss of this stem cell population and impaired regeneration after injury. ${ }^{81}$

Although some transcripts of activated and differentiated cells are detected in quiescent satellite cells, their proteins are generally detected after activation. As indicated above, MYOD is one such example where appearance of the protein identifies, and can promote satellite cell activation. In this context, repression of translation can be critical for maintaining satellite cell quiescence. This is mediated by phosphorylation of translation initiation factor elF2a, resulting in stabilisation of the quiescent state, as inhibition of elF2a phosphorylation results in exit from quiescence, activation of the myogenic programme and compromised selfrenewal. $^{82}$

Intriguingly, a decrease in the satellite cell pool can be associated ${ }^{40,46,51}$ or not ${ }^{48}$ with impaired regeneration capacity upon injury. Indeed, a recent report indicated that the dynamics and extent of regeneration, both at the stem cell population and histological levels, vary depending on the method used to induce muscle injury ${ }^{43}$ (snake venom myotoxins, chemicals or physical procedures). Furthermore, the number of satellite cells can increase several fold with some injury procedures raising further questions on how stem cell numbers are regulated locally, and globally. Other factors, such as the myofibre, can impact on satellite cell numbers. For example, transgenic mice overexpressing Tead1 (TEA domain transcription factor binds to Hippo signalling effectors YAP/TAZ) in myofibres have $6 \mathrm{X}$ more quiescent satellite cells. ${ }^{83}$ Although this transgenic mouse ameliorated muscle regeneration in a dystrophic $m d x$ background, it is not clear if satellite cell numbers have a role. Taken together, these reports raise the question-how many stem cells are required to assure proper muscle function during regeneration and ageing?

\section{Distinct states of quiescence}

Until recently, cellular quiescence was considered to be a passive and homogenous cellular state with reduced metabolic activity. Of note, recent reports indicate that quiescent satellite cells can exist in different states, more or less primed for commitment, ${ }^{84}$ or poised for activation. ${ }^{85}$ Satellite cells expressing high levels of Pax7 are less primed for commitment, display a lower metabolic activity and a delayed first mitosis upon activation in vivo and in vitro compared to cells expressing lower levels of Pax7. PAX7 ${ }^{\mathrm{Hi}}$ cells were therefore proposed to be in a deeper quiescent, or "dormant" cell state, compared to the remaining population that is more primed for cell-cycle entry. ${ }^{84}$ Hence, the quiescent state exists as a continuum from PAX7 ${ }^{\mathrm{Hi}}$ to PAX7 $7^{\text {Low }}$ cells.

During extreme stress, such as death, where cells are exposed to tissue necrosis, acidosis and lack of oxygen, the majority of satellite cells adopt a dormant cell state ${ }^{86}$ similar to that noted in a sub-population (PAX7 ${ }^{\mathrm{Hi}}$ ) in living mice. ${ }^{84}$ Therefore, satellite cells can modulate their physiological and metabolic status to adapt to changing microenvironments. It is likely that satellite cells that are primed would be first-responders to mild-injury, and that dormant 
Cellular Senescence

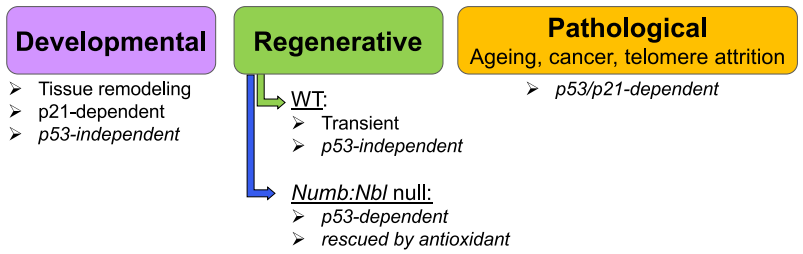

Fig. 4 Cellular senescence in different contexts. Although senescence has been extensively reported in pathological contexts ${ }^{90-92}$, recent studies have reported that cellular senescence is associated with developmental and regenerative processes, suggesting a beneficial role ${ }^{91,93-98}$. Senescence observed during muscle regeneration was not altered on a p53-null background, however, Numb: Numblike mutants that exhibit a higher level, and persistent cell senescence during regeneration, are rescued on a p53-null background and with antioxidants suggesting that senescence can be modulated differentially in this mutant compared to wild-type mice $^{93}$

satellite cells would be mobilised under severe trauma, however, this remains to be proven formally. By analogy, yeast that are subjected to nutrient deprivation alter their metabolic and transcriptome profiles significantly depending on the type of starvation regime employed. ${ }^{36}$ Therefore, one possibility is that dormancy vs. primed quiescence are determined by anatomical position. It is interesting to note that about $85 \%$ of satellite cells are located in close proximity to a blood vessel, whereas the remainder are distal, and likely in a more nutrient-deprived hypoxic niche, ${ }^{87}$ raising the possibility that these distally located cells might be in a dormant state.

Interestingly, quiescent satellite cells that are distant from a site of muscle injury (ex. contralateral muscle) can transit from G0 to the G0/G1 state (also called ' $G($ alert)'), characterised by an increased cell size, mitochondrial activity, gene expression profile, improved differentiation and enhanced regeneration capacity ${ }^{85}$ (Fig. 2a). Although the $\mathrm{BaCl}_{2}$ that was used to promote muscle injury could have diffused to distal muscles, some of the observed phenotypes were confirmed with physical injury suggesting a systemic relay mechanism. This $\mathrm{GO}-\mathrm{G}$ (alert) transition state is reversible and was shown to be dependent on the mTor signalling pathway. ${ }^{85}$ Taken together, these findings indicate that adult satellite cells exist in different quiescent states within which they can transit in response to environmental cues.

Following exit from quiescence, the fate a satellite cell can vary according to the context: apoptosis, ${ }^{40,51}$ direct differentiation ${ }^{46}$ or senescence. ${ }^{41,49}$ These cell states can be altered significantly during ageing, leading to a decline in satellite cell number and function ${ }^{39,49,88,89}$ as a result of defects in self-renewal, maintenance of quiescence, regenerative capacity or increased susceptibility to apoptosis and senescence. ${ }^{39,41,45,49}$

\section{DEREGULATION OF SATELLITE CELL FUNCTION: AGEING AND SENESCENCE}

Pathologies associated with ageing involve cell-autonomous as well as non-cell-autonomous mechanisms, including cellular senescence. Cellular senescence represents an irreversible cell cycle exit state that is often associated with pathologies where cancerous, DNAdamaged or ageing cells withdraw from the cell cycle and suppress apoptotic mechanisms while engaging tumour suppressors such as P53, P16/P19. ${ }^{90-92}$ An important signature of senescent cells that undergo a stress response such as oxidative, replicative and genotoxic stress, is the so-called "senescence-associated secretory phenotype" (SASP) where several growth factors, inflammatory molecules (chemokines, cytokines), proteases, and extracellular matrix components modify the fate of nearby cells. Currently, markers specific for senescent cells are lacking; commonly used markers include senescence-associated $\beta$-galactosidase (SA- $\beta$-Gal) activity in lysosomes, the level of lysosomal content, and elevated levels of P16. ${ }^{90,91}$ Although the pathological consequences of cellular senescence have been well documented where suppression of senescent cells can have dramatic consequences including increase of lifespan, its potential beneficial effects have only been reported recently for tissue remodelling during development and regeneration ${ }^{91,93-98}$ (Fig. 4). Suppression of senescence during prenatal stages does not promote overt phenotypes, however, removal of senescent endothelial and fibroblast cells was associated with less efficient wound healing of skin. ${ }^{97}$ These studies raise the notion of a beneficial role for cellular senescence, likely through the SASP (Fig. 4). Similarly, senescent cells were reported to have a role during limb regeneration in salamander following amputation. ${ }^{98}$ Furthermore, mutation of the endocytic adaptor proteins Numb: Numblike resulted in senescence of myogenic cells and compromised regeneration. This phenotype was rescued in a p53-null context, and by administration of an antioxidant. ${ }^{93}$ Notably, this Numb-mediated senescence was distinct from senescence observed in endothelial cells during early regeneration that was independent of $p 53$ and oxidative stress. ${ }^{93}$ It is interesting to note that cellular senescence also has a role in cell plasticity where the generation of teratomas following induction of pluripotency factors (Oct4, Sox2, $K I f 4$ and $M y c$ ) in vivo and ex vivo was modulated, at least in part, by senescent cells. ${ }^{95}$

Recent studies pointed to functional differences between aged and 'geriatric' mice where in the latter, the regenerative capacity of satellite cells decreases even further during extreme ageing, after 28 months of age in mice. Here, satellite cells lose their quiescent state to become pre-senescent, due to de-repression of the senescence marker p16 $16^{\text {INK } 4 a}$ following loss of the PRC1mediated repressive $\mathrm{H} 2 \mathrm{~A}$-lysine 119 ubiquitination mark. Upon injury, they fail to activate (absence of Myod expression) and transit to a full senescent state that is marked by increased p16 ${ }^{\text {INK4a }}$ expression, increased number of $\mathrm{\gamma}-\mathrm{H} 2 \mathrm{AX}$ foci indicating DNA double strand breaks, and appearance of a senescenceassociated $\beta$-galactosidase activity. Interestingly, this presenescent phenotype could be reverted by silencing of $p 16^{\text {INK4a }}$ but not by exposure to a youthful environment, underscoring the cell-autonomous nature of this pre-senescent phenotype. ${ }^{49}$ More recently, it was shown that aged satellite cells display decreased autophagy and mitophagy activities, leading to increased intracellular reactive-oxygen species. ${ }^{41}$ Notably, scavenging ROS restored $p 16^{I N K 4 a}$ repression by re-establishing its repressive H2Alysine 119 modification, decreased senescence and increased proliferation potential, while restoring autophagy reversed the senescence phenotype and restored the regenerative capacity of geriatric satellite cells. ${ }^{41,92}$ More generally, it appears that cellular quiescence protects satellite cells from ROS through upregulation of genes involved in the antioxidant response, such as Thioredoxin reductase 1, Sulfiredoxin and Glutathione peroxidase $3 .^{66}$

The FGF2/SPRY1 axis appears to be deregulated in aged muscles, yet it remains unclear how this signalling effects its action. In one study, maintenance of aged myofibres in basal medium resulted in compromised proliferation of satellite cells unless FGF2 was added exogenously. ${ }^{88}$ Satellite cells express FGF receptors 1 and 4, and FGFR1 appears as their functional FGF receptor, as Fgfr4 did not rescue satellite cell proliferation upon Fgfr1 deletion. ${ }^{99}$ Additional FGF ligands might be involved in the regulation of satellite cell proliferation in vivo, such as FGF6. ${ }^{100}$ In contrast, a decline in satellite cell numbers in the aged niche was attributed to increased FGF2 production and decreased Spry1 expression. ${ }^{39}$ Spry 1 is a tyrosine kinase receptor that is expressed in quiescent satellite cells, and it antagonises FGF2 signalling. Satellite cell deletion of Spry1 during regeneration prevents their return to quiescence, resulting in a decrease in the quiescent satellite cell pool after regeneration. ${ }^{48}$ Moreover, aged human 


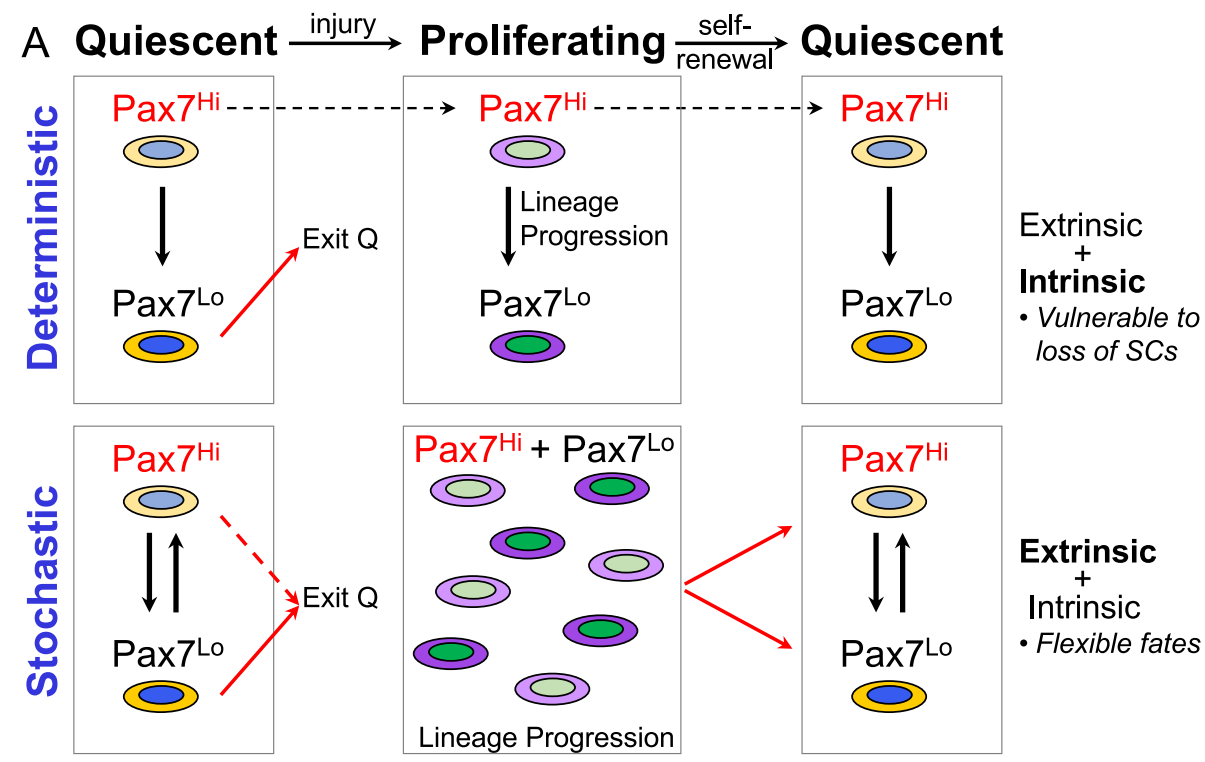

B
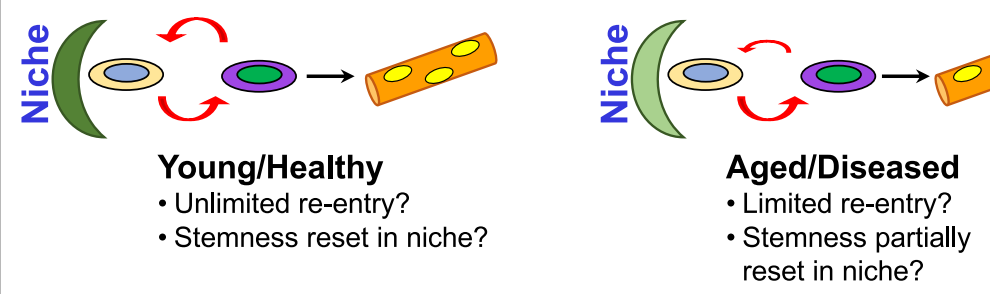

Fig. 5 Models for regulation of satellite cells during regeneration. a Deterministic model (top), where PAX7 ${ }^{\mathrm{Hi}}$ cells retain this state through proliferation and return to the quiescent state. Here PAX $7^{\mathrm{LO}}$ cells would derive from PAX $7^{\mathrm{Hi}}$ quiescent and proliferating cells and would be poised for commitment. This hierarchical model suggests intrinsic mechanisms as driving forces for maintenance of these relative states and would result in vulnerability if only a subset of the cells in the population have long-term stem-like properties. In the stochastic model (bottom), PAX7 $7^{\mathrm{Hi}}$ and $\mathrm{PAX} 7^{\mathrm{LO}}$ cells are interchangeable states, presumably due to fluctuations in gene expression, and obedience to extrinsic signals. This model proposes that all satellite cells have the potential to assume a stem-like or committed state. $\mathbf{b}$ Satellite cells undergo rounds of exit and entry into the niche to assume a quiescent state, and during ageing, exit from the niche is suggested to occur more frequently without replenishment, thereby resulting in declining numbers of muscle stem cells. We entertain the possibility that re-entry into the niche could reset or rejuvenate the stem cell and endow it with properties for long-term persistence

satellite cells exhibit higher levels of DNA methylation $(\mathrm{mC})$, and in this context, the SPRY1 promoter region was reported to have higher levels of $\mathrm{mC}$ compared to myogenic cells isolated from young individuals, thereby linking DNA methylation to increased sensitivity to higher levels of FGF2 in the aged niche. ${ }^{39,101}$ Impaired response to FGF2 signalling that results in elevated levels of $\mathrm{p} 38 \mathrm{a}$ and $\mathrm{p} 38 \beta$ diminishes self-renewal capacity of aged satellite cells, and pharmacological inhibition of this pathway, ${ }^{54,55}$ in combination with culture on soft hydrogel substrates ${ }^{55}$ leads to rescue of this phenotype.

Similarly, it has been observed that satellite cells attach to their surrounding microenvironment preferentially via fibronectin/ integrin interactions. Aged muscles display substantially lower levels of fibronectin, leading to a weaker adhesion of satellite cells and an increased susceptibility to anoikis, ${ }^{45}$ a phenotype similar to loss of $\beta 1$-integrin function in $\mathrm{SCs}^{47}$

Some of the decline in satellite cell function has also been attributed to deregulation of the Janus kinases (JAKs, via cytokine receptors), and Signal Transducer and Activator of Transcription proteins (STATs, relay of JAKs to activate nuclear targets) pathway, where STAT3 is stimulated by inflammatory cytokine associated with disease and ageing in muscle. Knockdown of Jak2 or Stat3 results in increased satellite stem cell divisions, and transient pharmacological inhibition of this pathway ameliorates transplantation potential and muscle force following injury. ${ }^{102,103}$

An apparent contradiction arises from the above-mentioned studies, regarding whether aged satellite cells can be rejuvenated in a non-cell-autonomous manner. Indeed, geriatric satellite cells could not reverse their pre-senescent state when transplanted into a young recipient muscle, ${ }^{49}$ whereas decreasing nichederived FGF activity ${ }^{39}$ or tissue complementation with exogenous fibronectin ${ }^{45}$ in aged muscles prevented the loss of satellite cells and restored a youthful muscle regeneration respectively. These findings suggest that the aged muscle phenotype can be reversed by exposure to a 'young' environment, however, the presenescent state of geriatric satellite cells might represent a point of no return. The most compelling evidence for youthful restoration, and the negative impact of circulating molecules in old mice, come from heterochronic parabiosis experiments, where young donor constituents (blood, secreted molecules, etc) transferred to an old host were shown to restore a rejuvenated phenotype in muscle and the nervous system. ${ }^{104,105}$ The identification of effector molecules that potentially mimic this response is an active area of research. The TGB $\beta$ member GDF11, which is homologous to Myostatin (GDF8, inhibitor of myogenesis), is one putative candidate, however its precise role remains under debate. ${ }^{106-108}$ 


\section{TO WHAT EXTENT IS THE MUSCLE STEM CELL POPULATION HETEROGENEOUS?}

Do proliferating and quiescent cell states each reflect a range of properties that individual myogenic cells can assume, or instead do myogenic cells represent distinct subpopulations each with fixed deterministic potential (Fig. 5)? In this context, it is interesting to note that heterogeneity has been suggested to be a feature of hematopoietic stem cells (HSCs) where at the top of the hierarchy, sub-classes of $\alpha$ and $\beta$ HSC subpopulations exist with equivalent myeloid potential, whereas a-HSCs have a reduced ability to produce mature lymphoid cells. ${ }^{109,110}$ Cellular plasticity can also underlie heterogeneity where endogenous cells are endowed with greater fate plasticity following their isolation and transplantation, for example, in the blood, skin, and muscle lineages. ${ }^{111-114}$

As indicated above, heterogeneity was also noted among quiescent satellite cells, suggesting that they can explore different levels of quiescence and metabolic states, perhaps driven by local niche signals. Our recent finding that satellite cells from different muscles exhibit different metabolic profiles ${ }^{115}$ suggests that at least some of the heterogeneities reported may be attributed to differences in myofibre niche properties.

Another feature of myogenic cells that is modulated during lineage progression is their response to DNA double strand induced stress. Recent findings have reported that adult stem cells are remarkably proficient in repairing DNA strand breaks compared to their committed progeny..$^{116-118}$ Specifically, satellite cells were reported to repair with greater efficiency and accuracy following irradiation-induced DNA damage, compared to myoblasts and differentiated cells, and that accurate repair depends on the key non-homologous end-joining factor DNA-PKcs (DNAdependent protein kinase, catalytic subunit). ${ }^{118}$ It remains to be determined if this resistance to DNA damage mediated stress persists in myopathies where satellite cells experience repeated bouts of quiescence and activation, or during ageing, and how this property affects mutational load and genome integrity over time.

Although heterogeneities have been reported among stem cell populations in different tissues, it remains unclear if this reflects a heterogeneity that is associated with distinct cell states, or if the population is uniform, but exhibits a wide range of behaviours. At the organismal level, individuals can survive extreme conditions of temperatures, dehydration and starvation, and some amphibians can undergo multiple consecutive freeze-thaw cycles with no apparent adverse effects. ${ }^{119}$ This can also be the case for individual cells thereby providing the opportunity to adapt to changing environments. However, empirical methods often do not expose these potentials. When cells are analysed, generally post-fixation, their properties several hours before or after the isolation procedure are eclipsed. Single cell transcriptomics can circumvent some of these shortcomings by documenting the range of behaviours in a population, as for example in intestinal and hematopoietic stem and committed cells. ${ }^{120,121}$

Heterogeneity among proliferating cells has been investigated to a limited extent. For example, myogenic cells perform symmetric (SCD) and asymmetric (ACD) cell divisions following muscle injury or after their isolation on single myofibres. ${ }^{35,40,84,122-129}$ It remains unclear what determines this choice, whether it is predetermined, and if SCD and ACD are obligate, or if switching in modes occurs in successive cell cycles. Several types of ACDs have been reported for muscle including non-random DNA segregation (NRDS), where old and new DNA strands segregate to distinct daughter cells, 35,40,84,122,128 transcription factors that include PAX7 (stem), MYOD (committed) and MYOGENIN (differentiated), 35,84,122,127 cytoplasmic proteins including NUMB, ${ }^{122,130} \mathrm{p38},{ }^{129}$ and Par proteins and dystrophin. ${ }^{125}$ In addition, several signalling pathways and extracellular molecules have been reported to influence the balance between SCD and ACD including fibronectin and WNT7a. ${ }^{126,131}$

It is clear that cell fates can be governed by extrinsic and intrinsic cues, and studies in Drosophila have shown the importance of extrinsic cues in neuroblast and germline cell divisions. ${ }^{35,132}$ Some insights also come from examining individual adult myogenic cells on micropatterns that represent artificial niches, where their shape can be designed to promote SCD and ACD. ${ }^{35,133,134}$ In this scenario, the frequency of NRDS and the asymmetric distribution of PAX7 and MYOGENIN were increased on fibronectin/fibrinogen coated micropatterns with an asymmetric motif compared to those that were placed on a symmetric motif. ${ }^{35}$ Therefore, extrinsic cell adhesion cues can have a major impact on at least two readouts for ACD. If these findings can be extrapolated to the in vivo situation, they suggest that the topology of the immediate microenvironment might dictate cell fate decisions in muscle.

However, these observations pose a conundrum. Although during muscle regeneration the microenvironment is highly dynamic, as far as cell types and extracellular matrix are concerned, a symmetric niche does not clearly exist in vivo; albeit it can be argued that a satellite cell undergoing planar cell divisions on a myofibre during homoeostasis might experience symmetrically distributed extrinsic cues. However, in absence of the myofibre, during G2/M when the axis of cell division will be determined, the resulting daughter cells will most likely experience distinct cues at opposite poles of the division axis in a regenerating tissue. If extrinsic cues have a predominant role in dictating cell fates, then one would expect that all divisions should be asymmetric - but this does not appear to be the case. How can in vitro and in vivo observations be reconciled? One possibility is that cells respond to threshold levels of extrinsic cues, and that an asymmetric response is triggered only if the differential in cell adhesion cues or signalling molecules at opposite poles of the division axis are sufficiently marked. Alternatively, intrinsic differences among cells might lend some to be more responsive to extrinsic asymmetric cues.

Beyond the notion of cellular heterogeneity, it is interesting to ask whether cellular memory persists over consecutive cell divisions. In other words, would a PAX7 ${ }^{\mathrm{Hi}}$ cell assume a PAX7 ${ }^{\mathrm{Hi}}$ cell state after one or several cycles of injury and return to homoeostasis, or alternatively, is the internal clock reset with each cell cycle thereby providing stochastic access to both self-renewal and commitment fates to each cell in the population (Fig. 5). Empirical evidence for cell equivalence is lacking in muscle, although this model is currently favoured for stem cells in other tissues. ${ }^{135-137}$

\section{MUSCLE REGENERATION-CAN IT BE IMPROVED?}

Altering genetic or epigenetic functions can lead to compromised regeneration raising the possibility that regeneration in wild-type mice might be improved if these processes can be manipulated. Regenerative myogenesis is non-uniform among mice of distinct genetic backgrounds; SJL/J mice regenerate faster than BALB/C mice following muscle injury or whole muscle engraftments. ${ }^{138}$ Moreover, a milder phenotype is observed with $M d x$ mice on a $129 / \mathrm{sv}$ background compared to DBA2 or C57BL/6 backgrounds. ${ }^{139,140}$ Nevertheless, dystrophic mice are generally less severely affected compared to the DMD condition in human. ${ }^{141}$

Strikingly, the MRL multi-strain mouse has been reported to be a "super-regenerator" compared to C57BL/6 for tissue recovery following an ear punch assay. ${ }^{142,143}$ When crossed with $\gamma^{-}$ Sarcoglycan null mouse, fibrosis and regeneration were reduced in skeletal and cardiac muscle, a phenotype that was mapped in part to a region on Chromosome $2 .{ }^{144}$ Metabolism was suggested to be a driver of this phenotype as the MRL mouse relies more on aerobic glycolytic energy metabolism, increased glutamate 
oxidation, and reduced fatty acid oxidation compared to C57BL/6 mice. $^{142}$

A notable example of how metabolism can impact disease is provided by the role of Nicotinamide adenine dinucleotide $\left(\mathrm{NAD}^{+}\right)$. Sirtuins consume $\mathrm{NAD}^{+}$and generate nicotinamide for deacetylation of proteins. Additionally, PARP (poly[adenosine $5^{\prime}$ diphosphate (ADP)-ribose] polymerase) proteins consume $\mathrm{NAD}^{+}$ during poly(ADP)-ribosylation of proteins. Interestingly, repletion of $\mathrm{NAD}^{+}$was shown to provide protection from metabolic diseases, mitochondrial dysfunction and necrosis, and resulted in improved skeletal and cardiac function in Dystrophin and Dystrophin:Utrophin mutant mice. ${ }^{145}$ This is thought to occur by countering increased PARP consumption of $\mathrm{NAD}^{+}$, thereby leading to the recovery of $\mathrm{NAD}^{+}$-dependent sirtuin signalling. In another study, treatment of mice with the $\mathrm{NAD}^{+}$precursor nicotinamide riboside was shown to induce the mitochondrial unfolded protein response, and this resulted in reduced senescence and increased lifespan in mice. ${ }^{146}$ Furthermore, satellite cells undergo a metabolic reprogramming upon in vitro activation after isolation, transiting from fatty acid oxidation to glycolysis as a main source of energy production. ${ }^{147}$ This is accompanied by decreased levels of $\mathrm{NAD}^{+}$, which directly regulates the activity of the histone deacetylase SIRT1. This in turn results in increased levels of histone acetylation and a subsequent activation of muscle gene transcription. Satellite cell-specific inactivation of Sirt 1 led to their precocious activation and differentiation. ${ }^{147}$

In another study, examination of foetal, perinatal, quiescent (young, post-mortem, aged) and regenerating myogenic stem cells identified striking differences in metabolic requirements. ${ }^{115}$ Specifically, aged satellite cells were shown to exhibit a compromised oxidative phosphorylation response, relying more on glycolysis for ATP production. In addition, proliferating foetal and perinatal myogenic cells have a low respiration demand, relying more on glycolysis compared to proliferating cells isolated from regenerating muscle, thereby underscoring the impact of the microenvironment on satellite cell metabolic response.

The AMP-activated protein kinase (AMPK) has several roles in metabolism including catabolism and activation of mitochondrial biogenesis. Interestingly, the key metabolic regulator AMPKa1 was shown to have a major role in controlling the balance between self-renewal and differentiation during muscle regeneration. ${ }^{148}$ Satellite cell-specific inactivation of AMPKa1 not only increased their glycolytic activity but also drastically enhanced their selfrenewal, leading to impaired muscle regeneration. This study places metabolism as a key parameter controlling satellite cell fate decisions.

As the metabolic activity of stem and progenitors is explored in more detail, it is important to note that this data is generally obtained from cells examined ex vivo, where culture conditions potentially alter metabolic profiles. Although relative comparisons have been highly informative, a key future objective is to generate in vivo readouts to assess directly the metabolic demands of stem and niche cells.

Reactive-oxygen species (ROS) have also been linked to a certain extent to pathological processes in regeneration. As indicated above the regeneration deficit when Numb:Numbl are conditionally inactivated in satellite cells can be rescued by administering the antioxidant $N$-acetyl cysteine (NAC). However, exposure to NAC does not improve the regenerative process in wild-type mice suggesting that oxidative stress is neither significantly promoting, nor deleterious to the regeneration process at early stages. ${ }^{93}$ During muscle differentiation, there is a higher demand for oxidative metabolism requiring mitochondrial biogenesis and increased ROS production. ${ }^{92}$ In another example, adult long-term-haematopoietic stem cells (LT-HSCs) use hypoxia induced glycolysis preferentially, ${ }^{149}$ perhaps to reduce ROS since these cells, as well as neural stem cells that do so through the action of FoxO, ${ }^{150}$ are sensitive to ROS levels that promote differentiation and apoptosis. It has been suggested that elevated levels of ROS drives stem cells out of quiescence in hypoxic conditions, and into proliferation when in normoxia, and that oxidative phosphoryation is low in LT-HSCs. ${ }^{151}$

Conditional inactivation of the transcription factors Pitx2:Pitx3 that act downstream of Pax3 and Pax7 results in elevated levels of ROS and compromised prenatal myogenesis, a phenotype that can be rescued by administration of NAC. ${ }^{152}$ The generation of ROS alone does not account for all of the oxidative stress. Indeed reactive nitrogen species (RNS; reaction of nitric oxide (NO) with superoxide $\left(\mathrm{O}_{2}^{-}\right)$to produce peroxynitrite $\left.\left(\mathrm{ONOO}^{-}\right)\right)$is often ignored when considering overall oxidative stress (combined ROS + RNS). The combined actions of ROS and RNS results in modifications of protein structure, lipids, signalling and cytoskeletal elements and DNA damage. $^{92}$

Autophagy is also intimately linked with metabolic processes. Here, damaged proteins and organelles are degraded by lysosomes to assure quality control. In conditions of limiting nutrients, as well as in quiescent cells, breakdown products resulting from autophagy can provide fuel for cellular activities. $^{41,92,153}$ It is clear that autophagy, as well as mitophagy, impact on cell and tissue response during stress in undamaged and injured muscles. ${ }^{41}$ In satellite cells, impairment of autophagy results in loss of proteostasis and increased oxidative stress due to mitochondrial dysfunction, resulting in senescence entry. ${ }^{41}$ These processes need to be examined in stromal cells to ascertain their global impact on muscle physiology.

In the context of myopathies where chronic cycles of degeneration and regeneration prevail, one key aim has been to improve the efficiency of the regeneration process. However, recent findings on Nfix mutant alone or on the myopathic $a$ Sarcoglycan null model showed that delaying the regeneration process and shifting to slow myogenesis has a dramatic positive outcome in functional tests including extensive running. ${ }^{154,155}$ Although Nfix deletion could act through other pathways unrelated to regeneration speed per se, these findings suggest that multiple pathways, including metabolic processes, could be targeted to temper rather than accelerate muscle regeneration.

\section{THE IMMUNE RESPONSE IN REGENERATION AND DISEASE}

The immune response during regeneration has become another central focus in recent years where monocytes, macrophages, neutrophils and $\mathrm{T}$ cells impact on the regeneration process. The critical role of macrophages in wound repair and muscle regeneration has been documented extensively. ${ }^{156} \mathrm{~A}$ proinflammatory response following production of macrophages from infiltrating monocytes, as well as resident macrophages, is associated with phagocytosis and clearing of debris in injured muscle. A second phase of inflammation that follows after a few days is associated with increased myofibre differentiation, angiogenesis, matrix remodelling and subsequently homoeostasis. The distinction between yolk sac and foetal liver derived macrophages raises the question of their respective roles and their dynamics during homoeostasis and regeneration. ${ }^{157,158}$ Furthermore, chronic myopathies are characterised by a nonsynchronous regeneration process resulting in the biphasic macrophage response being disrupted, and the coexistence of these populations contributing to the pathophysiology. ${ }^{156}$

Eosinophils and regulatory $\mathrm{T}$ cells also infiltrate the damaged muscle where they affect FAP and myogenic cell proliferation, respectively. ${ }^{159-161}$ Notably, regulatory $\mathrm{T}$ cells (Treg) produce Amphiregulin that was reported to improve the regeneration process by acting on myogenic cells. ${ }^{159}$ The inflammatory cytokine Prostaglandin E2 (PGE2) was also shown to stimulate satellite cell proliferation and enhance muscle regeneration by promoting the CAMP/phosphoCREB pathway and activation of the 
NURR1 transcription factor. ${ }^{162}$ Intriguingly, in addition to their promyogenic effects, restorative macrophages were shown to stimulate myogenesis/angiogenesis through secretion of Oncostatin $M,{ }^{163}$ whereas this compound is secreted by muscle fibres and functions to maintain satellite cell quiescence. ${ }^{81}$

As for myogenic cells, AMPKa1 has a key role in macrophages where it is required for the anti-inflammatory/restorative inflammatory phenotype during skeletal muscle regeneration. Loss of function in myeloid cells results in lasting inflammation and defects in muscle regeneration due to a block in the phenotypic transition of macrophages. ${ }^{161}$

In summary, recent studies on regenerative myogenesis have underscored the flexibility and wide range of responses exhibited by muscle satellite cells to a variety of stresses, by regulating their depth of quiescence, mode of proliferation and modulation of metabolic processes. Whether return to the quiescent state allows stem cells to reset their state, or perhaps even restore their potential, needs to be explored (Fig. 5b). As the study of quiescence takes more prominence, we note that the mirtron miR-708 was recently shown to regulate quiescence and self-renewal by antagonizing cell migration through targeting the transcripts of the focal-adhesion associated protein Tensin $3^{164}$. Thus, the G0 state exhibits multiple safeguards that merit future attention. Furthermore, the nature of the niche stromal cells is distinct spatially (ex. head vs. trunk) and temporally, pointing to a dynamic niche during prenatal life, adult, disease and ageing. These factors will likely have a significant impact on muscle stem cell properties, as well as their regenerative potential. Future research in these areas should provide valuable information on how to optimise regeneration and boost stem cell potential.

\section{ACKNOWLEDGEMENTS}

We acknowledge support from the Institut Pasteur, Association Française contre les Myopathies, Agence Nationale de la Recherche (Laboratoire d'Excellence Revive, Investissement d'Avenir; ANR-10-LABX-73), and the European Research Council (Advanced Research Grant 332893). We thank Marina Thizeau for illustrations of Figs. 1,2 , and $3 a$.

\section{AUTHOR CONTRIBUTIONS}

All authors researched, collated, and wrote this paper.

\section{ADDITIONAL INFORMATION}

Competing interests: The authors declare no competing interests.

Publisher's note: Springer Nature remains neutral with regard to jurisdictional claims in published maps and institutional affiliations.

\section{REFERENCES}

1. Tajbakhsh, S. Skeletal muscle stem cells in developmental versus regenerative myogenesis. J. Intern. Med. 266, 372-389 (2009).

2. Yin, H., Price, F. \& Rudnicki, M. A. Satellite cells and the muscle stem cell niche. Physiol. Rev. 93, 23-67 (2013).

3. Mauro, A. Satellite cell of skeletal muscle fibers. J. Biophys. Biochem. Cytol. 9, 493-495 (1961).

4. Comai, G. \& Tajbakhsh, S. Molecular and cellular regulation of skeletal myogenesis. Curr. Top. Dev. Biol. 110, 1-73 (2014).

5. Kassar-Duchossoy, L. et al. Mrf4 determines skeletal muscle identity in Myf5: Myod double-mutant mice. Nature 431, 466-471 (2004).

6. Rudnicki, M. A. et al. MyoD or myf-5 is required for the formation of skeletal muscle. Cell 75, 1351-1359 (1993).

7. Yamamoto, M. et al. Loss of MyoD and Myf5 in skeletal muscle stem cells results in altered myogenic programming and failed regeneration. Stem Cell Rep. 10, 956-969 (2018).

8. Gayraud-Morel, B. et al. Myf5 haploinsufficiency reveals distinct cell fate potentials for adult skeletal muscle stem cells. J. Cell Sci. 125, 1738-1749 (2012).
9. Sambasivan, R., Kuratani, S. \& Tajbakhsh, S. An eye on the head: the development and evolution of craniofacial muscles. Development 138, 2401-2415 (2011).

10. Kassar-Duchossoy, L. et al. Pax3/Pax7 mark a novel population of primitive myogenic cells during development. Genes Dev. 19, 1426-1431 (2005).

11. Relaix, F., Rocancourt, D., Mansouri, A. \& Buckingham, M. A Pax3/Pax7-dependent population of skeletal muscle progenitor cells. Nature 435, 948-953 (2005).

12. Sambasivan, R. et al. Distinct regulatory cascades govern extraocular and pharyngeal arch muscle progenitor cell fates. Dev. Cell. 16, 810-821 (2009).

13. Joe, A. W. et al. Muscle injury activates resident fibro/adipogenic progenitors that facilitate myogenesis. Nat. Cell Biol. 12, 153-163 (2010).

14. Uezumi, A., Fukada, S., Yamamoto, N., Takeda, S. \& Tsuchida, K. Mesenchymal progenitors distinct from satellite cells contribute to ectopic fat cell formation in skeletal muscle. Nat. Cell Biol. 12, 143-152 (2010).

15. Relaix, F. et al. Pw1/Peg3 is a potential cell death mediator and cooperates with Siah1a in p53-mediated apoptosis. Proc. Natl Acad. Sci. USA 97, 2105-2110 (2000).

16. Mitchell, K. J. et al. Identification and characterization of a non-satellite cell muscle resident progenitor during postnatal development. Nat. Cell Biol. 12, 257-266 (2010).

17. Dellavalle, A. et al. Pericytes of human skeletal muscle are myogenic precursors distinct from satellite cells. Nat. Cell Biol. 9, 255-267 (2007).

18. Bonfanti, C. et al. PW1/Peg3 expression regulates key properties that determine mesoangioblast stem cell competence. Nat. Commun. 6, 6364 (2015).

19. Liu, N. et al. A Twist2-dependent progenitor cell contributes to adult skeletal muscle. Nat. Cell Biol. 19, 202-213 (2017).

20. Lemos, D. R. et al. Nilotinib reduces muscle fibrosis in chronic muscle injury by promoting TNF-mediated apoptosis of fibro/adipogenic progenitors. Nat. Med. 21, 786-794 (2015)

21. Formicola, L. et al. Inhibition of the activin receptor type-2B pathway restores regenerative capacity in satellite cell-depleted skeletal muscle. Front. Physiol. 9, 515 (2018).

22. Sacchetti, B. et al. No identical "mesenchymal stem cells" at different times and sites: human committed progenitors of distinct origin and differentiation potential are incorporated as adventitial cells in microvessels. Stem Cell Rep. 6, 897-913 (2016).

23. Giordani, L. et al. High-dimensional single-cell cartography reveals novel skeletal muscle resident cell populations. BioRxiv 304683. https://doi.org/10.1101/ 304683 (2018).

24. Fiore, D. et al. Pharmacological blockage of fibro/adipogenic progenitor expansion and suppression of regenerative fibrogenesis is associated with impaired skeletal muscle regeneration. Stem Cell Res 17, 161-169 (2016).

25. Lepper, C., Partridge, T. A. \& Fan, C.-M. An absolute requirement for Pax7positive satellite cells in acute injury-induced skeletal muscle regeneration. Development 138, 3639-3646 (2011).

26. Murphy, M. M., Lawson, J. A., Mathew, S. J., Hutcheson, D. A. \& Kardon, G. Satellite cells, connective tissue fibroblasts and their interactions are crucial for muscle regeneration. Development 138, 3625-3637 (2011).

27. Sambasivan, R. et al. Pax7-expressing satellite cells are indispensable for adult skeletal muscle regeneration. Development 138, 3647-3656 (2011).

28. Ontell, M. \& Kozeka, K. The organogenesis of murine striated muscle: a cytoarchitectural study. Am. J. Anat. 171, 133-148 (1984).

29. Alonso-Martin, S. et al. Gene expression profiling of muscle stem cells identifies novel regulators of postnatal myogenesis. Front. Cell. Dev. Biol. 4, 58 (2016).

30. Mourikis, P., Gopalakrishnan, S., Sambasivan, R. \& Tajbakhsh, S. Cell-autonomous Notch activity maintains the temporal specification potential of skeletal muscle stem cells. Development 139, 4536-4548 (2012).

31. Pietrosemoli, N. et al. Comparison of multiple transcriptomes exposes unified and divergent features of quiescent and activated skeletal muscle stem cells. Skelet. Muscle 7, 28 (2017).

32. Yoshida, N., Yoshida, S., Koishi, K., Masuda, K. \& Nabeshima, Y. Cell heterogeneity upon myogenic differentiation: down-regulation of MyoD and Myf-5 generates 'reserve cells'. J. Cell Sci. 111, 769-779 (1998).

33. Monge, $C$. et al. Quiescence of human muscle stem cells is favored by culture on natural biopolymeric films. Stem Cell Res. Ther. 8, 104 (2017).

34. Quarta, M. et al. Bioengineered constructs combined with exercise enhance stem cell-mediated treatment of volumetric muscle loss. Nat. Commun. 8, 15613 (2017).

35. Yennek, S., Burute, M., Thery, M. \& Tajbakhsh, S. Cell adhesion geometry regulates non-random DNA segregation and asymmetric cell fates in mouse skeletal muscle stem cells. Cell Rep. 7, 961-970 (2014).

36. Klosinska, M. M., Crutchfield, C. A., Bradley, P. H., Rabinowitz, J. D. \& Broach, J. R. Yeast cells can access distinct quiescent states. Genes Dev. 25, 336-349 (2011).

37. Abou-Khalil, R. et al. Autocrine and paracrine angiopoietin $1 /$ Tie-2 signaling promotes muscle satellite cell self-renewal. Cell. Stem. Cell. 5, 298-309 (2009). 
38. Bjornson, C. R. et al. Notch signaling is necessary to maintain quiescence in adult muscle stem cells. Stem Cells 30, 232-242 (2012).

39. Chakkalakal, J. V., Jones, K. M., Basson, M. A. \& Brack, A. S. The aged niche disrupts muscle stem cell quiescence. Nature 490, 355-360 (2012).

40. Cheung, T. H. et al. Maintenance of muscle stem-cell quiescence by microRNA489. Nature 482, 524-528 (2012).

41. Garcia-Prat, L. et al. Autophagy maintains stemness by preventing senescence. Nature 529, 37-42 (2016).

42. Günther, S. et al. Myf5-positive satellite cells contribute to Pax7-dependent long-term maintenance of adult muscle stem cells. Cell. Stem Cell. https://doi. org/10.1016/j.stem.2013.07.016 (2013).

43. Hardy, D. et al. Comparative study of injury models for studying muscle regeneration in mice. PLOS ONE 11, e0147198 (2016).

44. Kitamoto, T. \& Hanaoka, K. Notch3 null mutation in mice causes muscle hyperplasia by repetitive muscle regeneration. Stem Cells 28, 2205-2216 (2010).

45. Lukjanenko, L. et al. Loss of fibronectin from the aged stem cell niche affects the regenerative capacity of skeletal muscle in mice. Nat. Med. 22, 897-905 (2016).

46. Mourikis, P. et al. A critical requirement for notch signaling in maintenance of the quiescent skeletal muscle stem cell state. Stem Cells 30, 243-252 (2012).

47. Rozo, M., Li, L. \& Fan, C. M. Targeting beta1-integrin signaling enhances regeneration in aged and dystrophic muscle in mice. Nat. Med. 22, 889-896 (2016).

48. Shea, K. L. et al. Sprouty1 regulates reversible quiescence of a self-renewing adult muscle stem cell pool during regeneration. Cell. Stem Cell. 6, 117-129 (2010).

49. Sousa-Victor, P. et al. Geriatric muscle stem cells switch reversible quiescence into senescence. Nature 506, 316-321 (2014).

50. von Maltzahn, J., Jones, A. E., Parks, R. J. \& Rudnicki, M. A. Pax7 is critical for the normal function of satellite cells in adult skeletal muscle. Proc. Natl Acad. Sci. USA. https://doi.org/10.1073/pnas.1307680110 (2013).

51. Yamaguchi, M. et al. Calcitonin receptor signaling inhibits muscle stem cells from escaping the quiescent state and the niche. Cell Rep. 13, 302-314 (2015).

52. Ikemoto, M. et al. Autologous transplantation of $\mathrm{SM} / \mathrm{C}-2.6(+)$ satellite cells transduced with micro-dystrophin CS1 cDNA by lentiviral vector into mdx mice. Mol. Ther. 15, 2178-2185 (2007).

53. Montarras, D. et al. Direct isolation of satellite cells for skeletal muscle regeneration. Science 309, 2064-2067 (2005).

54. Bernet, J. D. et al. p38 MAPK signaling underlies a cell-autonomous loss of stem cell self-renewal in skeletal muscle of aged mice. Nat. Med. https://doi.org/ 10.1038/nm.3465 (2014).

55. Cosgrove, B. D. et al. Rejuvenation of the muscle stem cell population restores strength to injured aged muscles. Nat. Med. https://doi.org/10.1038/nm.3464 (2014).

56. Gilbert, P. M. et al. Substrate elasticity regulates skeletal muscle stem cell selfrenewal in culture. Science 329, 1078-1081 (2010).

57. Chan, S. S. et al. Skeletal muscle stem cells from PSC-derived teratomas have functional regenerative capacity. Cell. Stem Cell. 23, 74-85 e76 (2018).

58. Fuchs, E. The tortoise and the hair: slow-cycling cells in the stem cell race. Cell 137, 811-819 (2009).

59. Li, L. \& Clevers, H. Coexistence of quiescent and active adult stem cells in mammals. Science 327, 542-545 (2010).

60. Coller, H. A., Sang, L. \& Roberts, J. M. A new description of cellular quiescence. PLoS Biol. 4, e83 (2006).

61. Dhawan, J. \& Laxman, S. Decoding the stem cell quiescence cycle-lessons from yeast for regenerative biology. J. Cell Sci. 128, 4467-4474 (2015).

62. Farina, N. H. et al. A role for RNA post-transcriptional regulation in satellite cell activation. Skelet. Muscle 2, 21 (2012)

63. Fukada, S. I. et al. Molecular signature of quiescent satellite cells in adult skeletal muscle. Stem Cells 1, 2448-2459 (2007).

64. Liu, L. et al. Chromatin modifications as determinants of muscle stem cell quiescence and chronological aging. Cell Rep. 4, 189-204 (2013).

65. Machado, L. et al. In situ fixation redefines quiescence and early activation of skeletal muscle stem cells. Cell Rep. 21, 1982-1993 (2017)

66. Pallafacchina, G. et al. An adult tissue-specific stem cell in its niche: a gene profiling analysis of in vivo quiescent and activated muscle satellite cells. Stem Cell Res. 4, 77-91 (2010).

67. van Velthoven, C. T. J., de Morree, A., Egner, I. M., Brett, J. O. \& Rando, T. A. Transcriptional profiling of quiescent muscle stem cells in vivo. Cell Rep. 21, 1994-2004 (2017).

68. Fukada, S. et al. Isolation, characterization, and molecular regulation of muscle stem cells. Front. Physiol. 4, 317 (2013).

69. Yamaguchi, M. et al. Calcitonin receptor and Odz4 are differently expressed in Pax7-positive cells during skeletal muscle regeneration. J. Mol. Histol. 43, 581-587 (2012).
70. Jones, N. C. et al. The p38alpha/beta MAPK functions as a molecular switch to activate the quiescent satellite cell. J. Cell Biol. 169, 105-116 (2005).

71. Lamph, W. W., Wamsley, P., Sassone-Corsi, P. \& Verma, I. M. Induction of protooncogene JUN/AP-1 by serum and TPA. Nature 334, 629-631 (1988).

72. Mourikis, P. \& Tajbakhsh, S. Distinct contextual roles for Notch signalling in skeletal muscle stem cells. BMC Dev. Biol. 14, 2 (2014).

73. Kim, J. H. et al. Sex hormones establish a reserve pool of adult muscle stem cells. Nat. Cell Biol. 18, 930-940 (2016).

74. Brohl, D. et al. Colonization of the satellite cell niche by skeletal muscle progenitor cells depends on Notch signals. Dev. Cell. 23, 469-481 (2012).

75. Wen, Y. et al. Constitutive Notch activation upregulates Pax7 and promotes the self-renewal of skeletal muscle satellite cells. Mol. Cell. Biol. 32, 2300-2311 (2012).

76. Bagutti, C., Forro, G., Ferralli, J., Rubin, B. \& Chiquet-Ehrismann, R. The intracellular domain of teneurin-2 has a nuclear function and represses zic-1-mediated transcription. J. Cell Sci. 116, 2957-2966 (2003).

77. Ishii, K. et al. Muscle satellite cell protein Teneurin-4 Regulates differentiation during muscle regeneration. Stem Cells 33, 3017-3027 (2015).

78. Suzuki, H. et al. Calcitonin-induced changes in the cytoskeleton are mediated by a signal pathway associated with protein kinase A in osteoclasts. Endocrinology 137, 4685-4690 (1996).

79. Baghdadi, M. B. et al. Reciprocal signalling by Notch-Collagen V-CALCR retains muscle stem cells in their niche. Nature 557, 714-718 (2018).

80. Sato, T., Yamamoto, T. \& Sehara-Fujisawa, A. miR-195/497 induce postnatal quiescence of skeletal muscle stem cells. Nat. Commun. 5, 4597 (2014).

81. Sampath, S. C. et al. Induction of muscle stem cell quiescence by the secreted niche factor Oncostatin M. Nat. Commun. 9, 1531 (2018).

82. Zismanov, V. et al. Phosphorylation of elF2alpha is a translational control mechanism regulating muscle stem cell quiescence and self-renewal. Cell. Stem Cell. 18, 79-90 (2016).

83. Southard, S., Kim, J. R., Low, S., Tsika, R. W. \& Lepper, C. Myofiber-specific TEAD1 overexpression drives satellite cell hyperplasia and counters pathological effects of dystrophin deficiency. Elife 5. https://doi.org/10.7554/eLife.15461 (2016).

84. Rocheteau, P., Gayraud-Morel, B., Siegl-Cachedenier, I., Blasco, M. A. \& Tajbakhsh, S. A subpopulation of adult skeletal muscle stem cells retains all template DNA strands after cell division. Cell 148, 112-125 (2012).

85. Rodgers, J. T. et al. mTORC 1 controls the adaptive transition of quiescent stem cells from G0 to G(Alert). Nature 510, 393-396 (2014).

86. Latil, M. et al. Skeletal muscle stem cells adopt a dormant cell state post mortem and retain regenerative capacity. Nat. Commun. 3, 903 (2012).

87. Christov, C. et al. Muscle satellite cells and endothelial cells: close neighbors and privileged partners. Mol. Biol. Cell. 4, 1397-1409 (2007).

88. Shefer, G., Van de Mark, D. P., Richardson, J. B. \& Yablonka-Reuveni, Z. Satellitecell pool size does matter: defining the myogenic potency of aging skeletal muscle. Dev. Biol. 294, 50-66 (2006).

89. Conboy, I. M., Conboy, M. J., Smythe, G. M. \& Rando, T. A. Notch-mediated restoration of regenerative potential to aged muscle. Science 302, 1575-1577 (2003).

90. He, S. \& Sharpless, N. E. Senescence in health and disease. Cell 169, 1000-1011 (2017).

91. Muñoz-Espín, D. et al. Programmed cell senescence during mammalian embryonic development. Cell 155, 1104-1118 (2013).

92. Ricchetti, M. How stem cells manage to escape senescence and ageing - while they can: a recent study reveals that autophagy is responsible for senescencedependent loss of regenerative potential of muscle stem cells during ageing. Bioessays 38, 857-862 (2016).

93. Le Roux, I., Konge, J., Le Cam, L., Flamant, P. \& Tajbakhsh, S. Numb is required to prevent p53-dependent senescence following skeletal muscle injury. Nat. Commun. 6, 8528 (2015).

94. Storer, M. et al. Senescence is a developmental mechanism that contributes to embryonic growth and patterning. Cell 155, 1119-1130 (2013).

95. Chiche, A. et al. Injury-induced senescence enables in vivo reprogramming in skeletal muscle. Cell. Stem. Cell. 20, 407-414.e404 (2017).

96. Chang, J. et al. Clearance of senescent cells by ABT263 rejuvenates aged hematopoietic stem cells in mice. Nat. Med. 22, 78-83 (2016).

97. Demaria, M. et al. An essential role for senescent cells in optimal wound healing through secretion of PDGF-AA. Dev. Cell. 31, 722-733 (2014).

98. Yun, M. H., Davaapil, H. \& Brockes, J. P. Recurrent turnover of senescent cells during regeneration of a complex structure. Elife 4. https://doi.org/10.7554/ eLife.05505 (2015)

99. Yablonka-Reuveni, Z., Danoviz, M. E., Phelps, M. \& Stuelsatz, P. Myogenic-specific ablation of Fgfr1 impairs FGF2-mediated proliferation of satellite cells at the myofiber niche but does not abolish the capacity for muscle regeneration. Front Aging Neurosci. 7, 85 (2015). 
100. Kastner, S., Elias, M. C., Rivera, A. J. \& Yablonka-Reuveni, Z. Gene expression patterns of the fibroblast growth factors and their receptors during myogenesis of rat satellite cells. J. Histochem. Cytochem. 48, 1079-1096 (2000).

101. Bigot, A. et al. Age-associated methylation suppresses SPRY1, leading to a failure of re-quiescence and loss of the reserve stem cell pool in elderly muscle. Cell Rep. 13, 1172-1182 (2015).

102. Price, F. D. et al. Inhibition of JAK-STAT signaling stimulates adult satellite cell function. Nat. Med. 20, 1174-1181 (2014).

103. Tierney, M. T. et al. STAT3 signaling controls satellite cell expansion and skeletal muscle repair. Nat. Med. 20, 1182-1186 (2014).

104. Brack, A. S. et al. Increased Wnt signaling during aging alters muscle stem cell fate and increases fibrosis. Science 317, 807-810 (2007).

105. Conboy, I. M. et al. Rejuvenation of aged progenitor cells by exposure to a young systemic environment. Nature 433, 760-764 (2005).

106. Egerman, M. A. et al. GDF11 increases with age and inhibits skeletal muscle regeneration. Cell. Metab. 22, 164-174 (2015).

107. Hinken, A. C. et al. Lack of evidence for GDF11 as a rejuvenator of aged skeletal muscle satellite cells. Aging Cell. 15, 582-584 (2016).

108. Sinha, M. et al. Restoring systemic GDF11 levels reverses age-related dysfunction in mouse skeletal muscle. Science 344, 649-652 (2014).

109. Benz, C. et al. Hematopoietic stem cell subtypes expand differentially during development and display distinct lymphopoietic programs. Cell. Stem Cell. 10, 273-283 (2012).

110. Luchsinger, L. L., de Almeida, M. J., Corrigan, D. J., Mumau, M. \& Snoeck, H. W. Mitofusin 2 maintains haematopoietic stem cells with extensive lymphoid potential. Nature 529, 528-531 (2016).

111. Driskell, R. R. et al. Distinct fibroblast lineages determine dermal architecture in skin development and repair. Nature 504, 277-281 (2013).

112. Gayraud-Morel, B. et al. A role for the myogenic determination gene Myf5 in adult regenerative myogenesis. Dev. Biol. 312, 13-28 (2007).

113. Jensen, K. B. et al. Lrig1 expression defines a distinct multipotent stem cell population in mammalian epidermis. Cell. Stem Cell. 4, 427-439 (2009).

114. Sun, J. et al. Clonal dynamics of native haematopoiesis. Nature 514, 322-327 (2014).

115. Pala, F. et al. Distinct metabolic states govern skeletal muscle stem cell fates during prenatal and postnatal myogenesis. J. Cell. Sci. 131. https://doi.org/ 10.1242/jcs.212977 (2018)

116. Mohrin, M. et al. Hematopoietic stem cell quiescence promotes error-prone DNA repair and mutagenesis. Cell. Stem Cell. 7, 174-185 (2010).

117. Sotiropoulou, P. A. et al. Bcl-2 and accelerated DNA repair mediates resistance of hair follicle bulge stem cells to DNA-damage-induced cell death. Nat. Cell Biol. 12, 572-582 (2010)

118. Vahidi Ferdousi, L. et al. More efficient repair of DNA double-strand breaks in skeletal muscle stem cells compared to their committed progeny. Stem Cell Res 13, 492-507 (2014).

119. Storey, K. B. \& Storey, J. M. Molecular physiology of freeze tolerance in vertebrates. Physiol. Rev. 97, 623-665 (2017).

120. Grun, D. et al. De novo prediction of stem cell identity using single-cell transcriptome data. Cell. Stem Cell. 19, 266-277 (2016).

121. Nestorowa, S. et al. A single-cell resolution map of mouse hematopoietic stem and progenitor cell differentiation. Blood 128, e20-e31 (2016).

122. Shinin, V., Gayraud-Morel, B., Gomes, D. \& Tajbakhsh, S. Asymmetric division and cosegregation of template DNA strands in adult muscle satellite cells. Nat. Cell Biol. 8, 677-682 (2006)

123. Evano, B. \& Tajbakhsh, S. Sorting DNA with asymmetry: a new player in gene regulation? Chromosome Res. 21, 225-242 (2013).

124. Kuang, S., Kuroda, K., Le Grand, F. \& Rudnicki, M. A. Asymmetric self-renewal and commitment of satellite stem cells in muscle. Cell 129, 999-1010 (2007).

125. Dumont, N. A. et al. Dystrophin expression in muscle stem cells regulates their polarity and asymmetric division. Nat. Med. 21, 1455-1463 (2015).

126. Le Grand, F., Jones, A. E., Seale, V., Scime, A. \& Rudnicki, M. A. Wnt7a activates the planar cell polarity pathway to drive the symmetric expansion of satellite stem cells. Cell. Stem Cell. 4, 535-547 (2009).

127. Liu, W. et al. Hypoxia promotes satellite cell self-renewal and enhances the efficiency of myoblast transplantation. Development 139, 2857-2865 (2012).

128. Conboy, M. J., Karasov, A. O. \& Rando, T. A. High incidence of non-random template strand segregation and asymmetric fate determination in dividing stem cells and their progeny. PLoS Biol. 5, e102 (2007).

129. Troy, A. et al. Coordination of satellite cell activation and self-renewal by parcomplex-dependent asymmetric activation of p38a/ $\beta$ MAPK. Cell. Stem Cell. 11, 541-553 (2012).

130. Conboy, I. M. \& Rando, T. A. The regulation of Notch signaling controls satellite cell activation and cell fate determination in postnatal myogenesis. Dev. Cell. 3, 397-409 (2002).
131. Bentzinger, C. F. et al. Fibronectin regulates wnt7a signaling and satellite cell expansion. Cell. Stem Cell. 12, 75-87 (2013).

132. Neumüller, R. A. \& Knoblich, J. A. Dividing cellular asymmetry: asymmetric cell division and its implications for stem cells and cancer. Genes Dev. 23, 2675-2699 (2009).

133. Théry, M., Jimenez-Dalmaroni, A., Racine, V., Bornens, M. \& Julicher, F. Experimental and theoretical study of mitotic spindle orientation. Nature 447, 493-496 (2007).

134. Vignaud, T., Blanchoin, L. \& Théry, M. Directed cytoskeleton self-organization. Trends Cell. Biol. https://doi.org/10.1016/j.tcb.2012.08.012 (2012).

135. Blanpain, C. \& Simons, B. D. Unravelling stem cell dynamics by lineage tracing. Nat. Rev. Mol. Cell Biol. 14, 489-502 (2013).

136. Mascré, G. et al. Distinct contribution of stem and progenitor cells to epidermal maintenance. Nature 489, 257-262 (2012).

137. Snippert, H. J. et al. Intestinal crypt homeostasis results from neutral competition between symmetrically dividing Lgr5 stem cells. Cell 143, 134-144 (2010).

138. Roberts, P., McGeachie, J. K. \& Grounds, M. D. The host environment determines strain-specific differences in the timing of skeletal muscle regeneration: crosstransplantation studies between $\mathrm{SJL} / \mathrm{J}$ and BALB/C mice. J. Anat. 191( $\mathrm{Pt} 4)$, 585-594 (1997).

139. Calyjur, P. C. et al. The mdx mutation in the $129 / \mathrm{Sv}$ background results in a milder phenotype: transcriptome comparative analysis searching for the protective factors. PLOS ONE 11, e0150748 (2016).

140. Rodrigues, M. et al. Impaired regenerative capacity and lower revertant fibre expansion in dystrophin-deficient mdx muscles on DBA/2 background. Sci. Rep. 6, 38371 (2016).

141. Duddy, W. et al. Muscular dystrophy in the $\mathrm{mdx}$ mouse is a severe myopathy compounded by hypotrophy, hypertrophy and hyperplasia. Skelet. Muscle 5, 16 (2015).

142. Naviaux, R. K. et al. Retained features of embryonic metabolism in the adult MRL mouse. Mol. Genet. Metab. 96, 133-144 (2009).

143. Zhang, Y. et al. Drug-induced regeneration in adult mice. Sci. Transl. Med 7, 290ra292 (2015)

144. Heydemann, A. et al. The superhealing MRL background improves muscular dystrophy. Skelet. Muscle 2, 26 (2012).

145. Ryu, D. et al. NAD ${ }^{+}$repletion improves muscle function in muscular dystrophy and counters global PARylation. Sci. Transl. Med 8, 361ra139 (2016).

146. Zhang, $\mathrm{H}$. et al. $\mathrm{NAD}(+)$ repletion improves mitochondrial and stem cell function and enhances life span in mice. Science 352, 1436-1443 (2016).

147. Ryall, J. G. et al. The NAD(+)-dependent SIRT1 deacetylase translates a metabolic switch into regulatory epigenetics in skeletal muscle stem cells. Cell. Stem Cell. 16, 171-183 (2015).

148. Theret, M. et al. AMPKalpha1-LDH pathway regulates muscle stem cell selfrenewal by controlling metabolic homeostasis. EMBO J. 36, 1946-1962 (2017).

149. Takubo, K. et al. Regulation of glycolysis by Pdk functions as a metabolic checkpoint for cell cycle quiescence in hematopoietic stem cells. Cell. Stem Cell. 12, 49-61 (2013)

150. Yeo, $\mathrm{H}$. et al. FoxO3 coordinates metabolic pathways to maintain redox balance in neural stem cells. EMBO J. 32, 2589-2602 (2013).

151. Shyh-Chang, N., Daley, G. Q. \& Cantley, L. C. Stem cell metabolism in tissue development and aging. Development 140, 2535-2547 (2013).

152. L'Honore, A. et al. Redox regulation by Pitx2 and Pitx3 is critical for fetal myogenesis. Dev. Cell. 29, 392-405 (2014).

153. Anding, A. L. \& Baehrecke, E. H. Cleaning house: selective autophagy of organelles. Dev. Cell. 41, 10-22 (2017).

154. Rossi, G. et al. Nfix regulates temporal progression of muscle regeneration through modulation of myostatin expression. Cell Rep. 14, 2238-2249 (2016).

155. Rossi, G. et al. Silencing Nfix rescues muscular dystrophy by delaying muscle regeneration. Nat. Commun. 8, 1055 (2017).

156. Juban, G. \& Chazaud, B. Metabolic regulation of macrophages during tissue repair: insights from skeletal muscle regeneration. FEBS Lett. 591, 3007-3021 (2017).

157. Ginhoux, F. \& Guilliams, M. Tissue-resident macrophage ontogeny and homeostasis. Immunity 44, 439-449 (2016).

158. Gomez Perdiguero, E. et al. Tissue-resident macrophages originate from yolksac-derived erythro-myeloid progenitors. Nature 518, 547-551 (2015).

159. Burzyn, D. et al. A special population of regulatory $T$ cells potentiates muscle repair. Cell 155, 1282-1295 (2013).

160. Heredia, J. E. et al. Type 2 innate signals stimulate fibro/adipogenic progenitors to facilitate muscle regeneration. Cell 153, 376-388 (2013).

161. Mounier, R. et al. AMPKalpha1 regulates macrophage skewing at the time of resolution of inflammation during skeletal muscle regeneration. Cell. Metab. 18, 251-264 (2013). 
162. Ho, A. T. V. et al. Prostaglandin E2 is essential for efficacious skeletal muscle stem-cell function, augmenting regeneration and strength. Proc. Natl. Acad. Sci. USA 114, 6675-6684 (2017).

163. Latroche, C. et al. Coupling between myogenesis and angiogenesis during skeletal muscle regeneration is stimulated by restorative macrophages. Stem Cell Rep. 9, 2018-2033 (2017).

164. Baghdadi, M. B. et al. Notch-Induced miR-708 antagonizes satellite cell migration and maintains quiescence. Cell Stem Cell 23, 859-868.e5 (2018).

Open Access This article is licensed under a Creative Commons Attribution 4.0 International License, which permits use, sharing, adaptation, distribution and reproduction in any medium or format, as long as you give appropriate credit to the original author(s) and the source, provide a link to the Creative Commons license, and indicate if changes were made. The images or other third party material in this article are included in the article's Creative Commons license, unless indicated otherwise in a credit line to the material. If material is not included in the article's Creative Commons license and your intended use is not permitted by statutory regulation or exceeds the permitted use, you will need to obtain permission directly from the copyright holder. To view a copy of this license, visit http://creativecommons. org/licenses/by/4.0/.

(c) The Author(s) 2018 